\title{
ENXERTO AUTÓGENO DE CRISTA ILÍACA NA RECONSTRUÇÃO DO PROCESSO ALVEOLAR EM PORTADORES DE FISSURA LABIOPALATINA - ESTUDO DE 30 CASOS
}

\section{ILIAC CREST AUTOGENOUS GRAFT IN RECONSTRUCTION OF ALVEOLAR PROCESS IN CLEFT PATIENTS - STUDY OF 30 CASES}

\author{
João Luiz Carlini ${ }^{1}$ \\ André Luís Zétola ${ }^{2}$ \\ Ricardo Pires de Souza ${ }^{1}$ \\ Odilon Victor Porto Denardin ${ }^{3}$ \\ Abrão Rapoport, TCBC-SP ${ }^{4}$
}

\begin{abstract}
RESUMO: A reconstrução do processo alveolar em pacientes portadores de fissuras labiopalatais (FLP) é fundamental para erupção de dentes permanentes e para o restabelecimento estético através de implantes osteointegrados. Assim, considerado o alto grau de reabsorção do enxerto de osso autógeno puro e triturado, optou-se no Centro de Atendimento Integral ao Fissurado Labiopalatino, Curitiba, Paraná (1997 a 1999), pelo estabelecimento de dois grupos de 15 pacientes. No primeiro, foi utilizada crista ilíaca triturada e no outro combinada com hidroxiapatita biorreabsorvível. Ao fim de seis meses, os resultados cirúrgicos foram avaliados através de radiografia periapical digital, determinando quatro linhas limites para a reconstrução óssea. A primeira linha foi traçada na região cervical do dente adjacente à fissura que apresentava menor distorção. As outras linhas foram traçadas paralelamente à linha 1 , distando $3 \mathrm{~mm}$ entre cada uma delas. O espaço entre as linhas $1 \mathrm{e} 2$ foi denominado de $\mathrm{A}$, entre 2 e 3 de B e 3 e 4 de C. Para critérios de avaliação, quando o enxerto permaneceu no espaço A, foi considerado de sucesso, em contraposição aos espaços $\mathrm{B}$ e $\mathrm{C}$, quando foram denominados de procedimentos malsucedidos. Como resultados finais, o espaço A foi constatado em $46,7 \%$ dos pacientes submetidos ao procedimento controle com crista ilíaca e $80 \%$ para o grupo experimental, quando o enxerto ósseo foi empregado conjuntamente com o material aloplástico (hidroxiapatita).
\end{abstract}

Descritores: Enxerto; Osso; Autógeno.

\section{INTRODUÇÃO}

As fissuras labiopalatinas (FLP) são consideradas as alterações congênitas de face com maior incidência mundial, sendo que no Brasil ocorreu na proporção de 1:650 nascimentos, havendo uma estimativa de 225.000 portadores dessas lesões no país. A frequiência das FLP é maior em orientais e menor em negros, sendo a relação homem/ mulher de 3:2 (a estrutura mais afetada no homem é o lábio e na mulher o palato), sendo que $3 / 4$ dos pacientes afetados apresentam fendas unilaterais e $1 / 4$ bilaterais ${ }^{1,2}$.
Quanto à classificação das FLP, empregamos a de Spina $^{3}$, que, adotando como referência o forame incisivo, separa as fissuras em pré, trans, pós-incisivos.

A grande discussão sobre a reparação cirúrgica das FLP foi estabelecida quanto ao momento de sua indicação. Inicialmente, segundo Witsenburg ${ }^{4}$, promovia-se o enxerto na região do defeito no processo alveolar no mesmo tempo cirúrgico do fechamento do lábio e do palato, causando, todavia, uma deficiência no desenvolvimento da maxila. Porém, Boyne e Sands ${ }^{5}$, recomendavam como a idade ideal para realização do enxerto dos nove aos 12 anos,

1. Mestres em Cirurgia de Cabeça e Pescoço do Hospital Heliópolis, Hosphel, SP.

2. Mestrando em Cirurgia de Cabeça e Pescoço do Hospital Heliópolis, Hosphel, SP.

3. Doutor em Medicina pela UNIFESP.

4. Coordenador do CPG, Hosphel, SP.

Recebido em 21/2/2000

Aceito para publicação em 5/9/2000

Trabalho realizado no Serviço de Cirurgia de Cabeça e Pescoço do Hospital Heliópolis - Hosphel - São Paulo-SP. 
antes da erupção do canino na FLP. Propiciava-se com isto, o posicionamento correto dos dentes permanentes no processo alveolar reconstruído, ao lado do movimento dos dentes pela área fissurada através do tratamento ortodôntico e da restauração da estética facial pela relocação da prótese.

A escolha do enxerto ósseo autógeno puro e triturado foi determinada pelo atendimento dos requisitos de biocompatibilidade. Todavia, o seu grau de reabsorção justificou o emprego de material aloplástico (hidroxiapatita), que garantia uma boa osteocondução quando utilizada a crista ilíaca ${ }^{6}$.

\section{MÉTODOS}

No Centro de Cirurgia Buco-Maxilo-Facial do Centro de Atendimento Integrado de Fissurados-CAIF, Curitiba, Paraná, de agosto de 1997 a agosto de 1999, foram tratados cirurgicamente 30 pacientes com FLP através de enxerto autógeno de crista ilíaca para a reconstrução do processo alveolar.

No grupo controle (A), 15 foram submetidos a enxerto de crista ilíaca e no grupo experimental (B), outros 15 ao mesmo procedimento, todavia, em associação com material aloplástico (hidroxiapatita).

Para a seleção de pacientes, optamos por:

1. Todos deveriam ter de nove a 12 anos, idade ideal para o enxerto.

2. Preferência para pacientes moradores de Curitiba (facilidade de acompanhamento).

3. Prioridade aos portadores de FLP unilaterais em relação às bilaterais (melhor controle radiológico e índices de complicação mais baixo).

\section{No CAIFos}

Pacientes são divididos em grupo controle (A) e experimental (B) por ordem de chegada à Instituição.

Existe equipe multidisciplinar em atividades conjunta (psicólogos, assistentes sociais, cirurgiões buco-maxilo-faciais, ortodentistas, cirurgiões plásticos, otorrinolaringologistas, pediatras e fonoaudiólogos).

A cronologia de atuação é a seguinte:

- 3 meses: correção da fenda labial

- 18 meses: correção da fenda palatina

- 6 anos: início do tratamento ortodôntico

- 9 aos 12 anos: tratamento cirúrgico

As cirurgias foram realizadas sob anestesia geral, com intubação nasotraqueal contralateral à FLP, sendo utilizado como vasoconstritor local a lidocaína a $2 \%$, com adrenalina diluída a 1:200.000, após anti-sepsia com iodopolivinil pirrolidina (IPP). A seguir passou-se à individualização de retalho mucoperiostal de fundo de saco gengivolabial e jugal (até o primeiro molar), com incisões relaxantes entre os prémolares (Figura 1). Praticou-se a divisão do retalho mucoso em uma porção superior para reconstrução nasal (sutura com poligalactina 5-0) e uma inferior para fechamento palatino (sutura com poligalactina 4.0). Para refazer o assoalho ósseo da fossa nasal, foi utilizado um fragmento ósseo de cris- ta ilíaca (porção cortical triturada), não sendo utilizado nenhum método de fixação, promovendo-se a separação definitiva das cavidades nasal e bucal (Figura 2). Em seguida, utilizou-se a porção medular do fragmento de crista ilíaca individualizado, de forma triturada pura ou combinada com material aloplástico (hidroxiapatita) para preenchimento da fenda palatina existente (Figura 3).

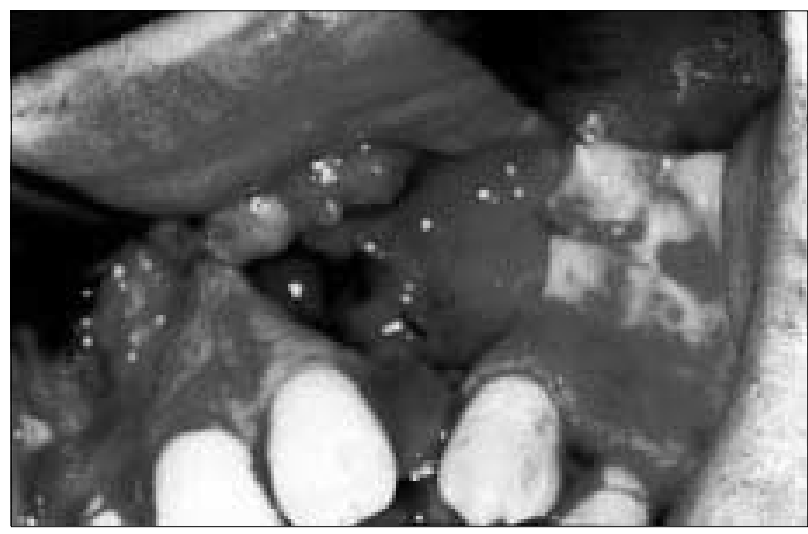

Figura 1 - Acesso cirúrgico ao defeito ósseo da fissura no processo alveolar.

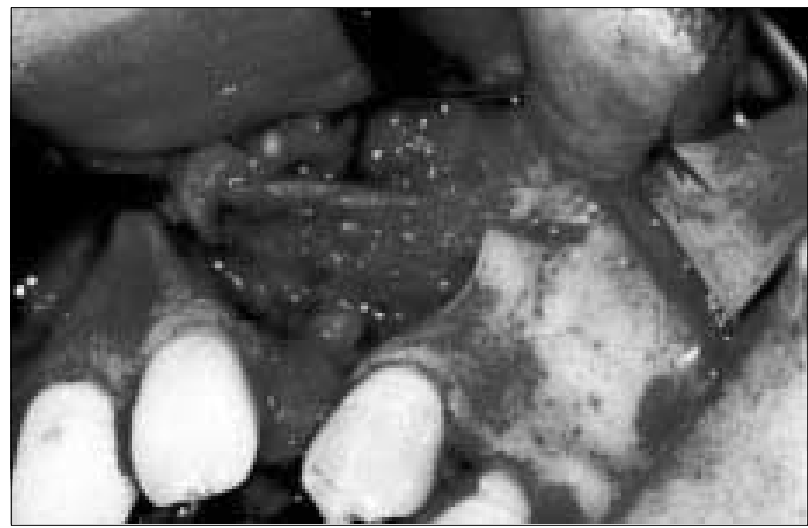

Figura 2 - Colocação da lâmina óssea para reconstrução do assoalho da fossa nasal.

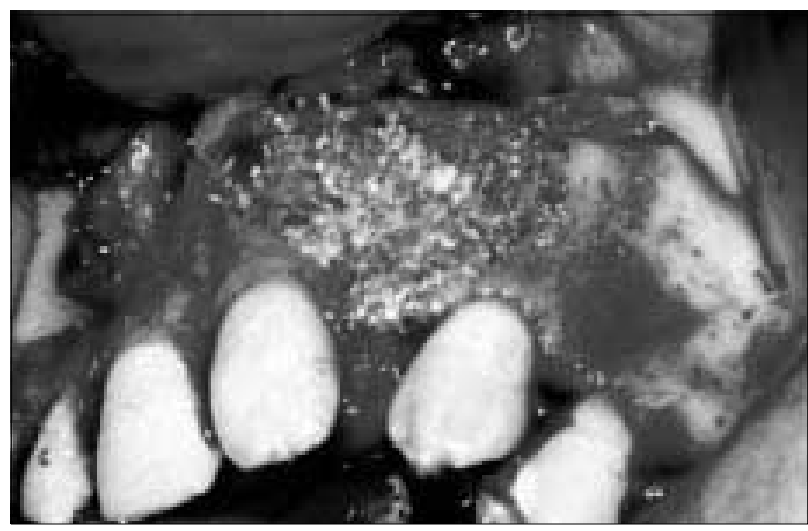

Figura 3 - Osso autógeno triturado combinado com hidroxiapatita, preenchendo o defeito ósseo do processo alveolar. 
Para individualização de fragmento de crista ilíaca, após anti-sepsia local com IPP, foi demarcada a área doadora e, através de incisão de $4 \mathrm{~cm}$, para retirada de porção cortical utilizada na reparação do soalho nasal, e da medular triturada para preenchimento do defeito palatino. Ao final, a área doadora é sintetizada com fios reabsorvíveis 3.0 (plano muscular e fáscia) e 4.0 para a pele, não ocorrendo complicações nesta área.

\section{Avaliação Através de Radiografia Digital}

Após quatro a seis meses do procedimento cirúrgico, é feita com tempo de exposição de 0,16 s, sendo utilizado sensor com magnitude do em torno de $2 \%$. A preferência do uso do método foi decorrente da melhor evidência da densidade e nitidez, menor dose de irradiação, ajuste do controle das imagens, informação instantânea, melhor avaliação das bordas para mensuração e possibilidade de sobrepor gráficos sobre as imagens para avaliação. Estas imagens, captadas pelo sensor, são projetadas na tela do computador onde foi criada uma escala numérica proporcional ao tratamento da imagem real na proporção de 21 pixels $/ \mathrm{mm}$. A partir desta escala, traçamos quatro retas paralelas para avaliar os resultados dos enxertos (Figura 4).

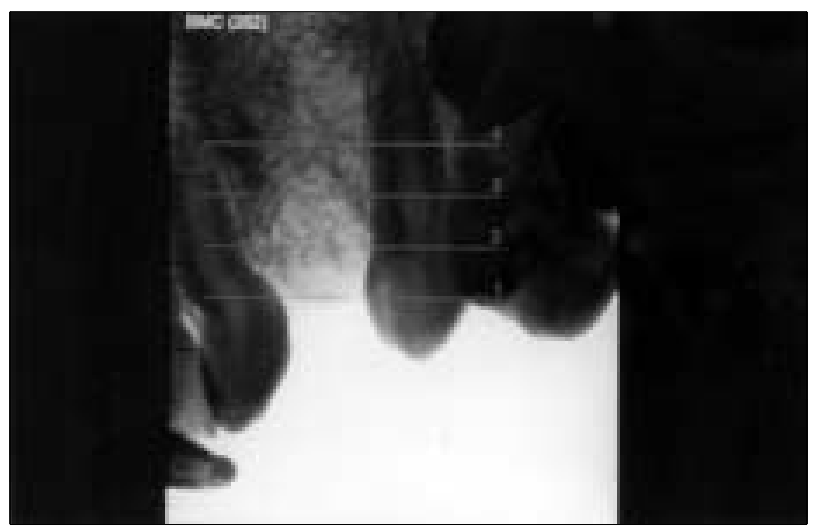

Figura 4 - Avaliação radiográfica da área enxertada, com as linhas que demarcam as áreas.Área $A$ - sucesso entre as linhas 1 e 2. Área $B-$ sucesso parcial entre as linhas 2 e 3 . Área $C-$ insucesso entre as linhas 3 e 4.

\section{Determinação de Linhas e dos Espaços à Radiografia Digital}

- Linha 1: traçada na região cervical do dente adjacente à fissura, que apresenta menor distorção.

- Linhas 2, 3 e 4: paralelas à linha 1 , distando $3 \mathrm{~mm}$ entre cada uma delas. (foto ou esquema)

- Espaço A: maior cavidade do enxerto entre as linhas 1 e 2.

- Espaço B: maior cavidade do enxerto entre as linhas 2 e 3.

- Espaço C: maior cavidade do enxerto entre as linhas 3 e 4.
Enxertos ósseos autógenos são utilizados por promoverem a indução óssea, favorecendo a osteogênese em combinação com a hidroxiapatita (HAP-91), biomaterial sintetizado a partir do calcário, que permite o crescimento ósseo dentro de sua estrutura e conseqüente regeneração a partir dos 60 dias.

Com a finalidade de modelar a probabilidade teórica dos enxertos ocuparem ou não o espaço A, utilizou-se a regressão logística, sendo definida ocupação do espaço A sob a influência da idade e das duas terapêuticas empregadas no modelo ajustado.

\section{RESULTADOS}

$\mathrm{Na}$ avaliação dos resultados, foram aferidos quatro parâmetros para os grupos A (enxerto ósseo) e B (enxerto ósseo + hidroxiapatita) - Quadro 1

\section{Quadro 1}

Variáveis demográficas, anatômicas e radiológicas aferidas nas fissuras labiopalatinas

\begin{tabular}{lcc}
\hline & Grupo A & Grupo B \\
\hline $\begin{array}{l}\text { Idade } \\
(9-12 \text { anos) } \\
(>12 \text { anos })\end{array}$ & $6(40,0 \%)$ & $7(46,7 \%)$ \\
\hline Sexo & $9(60,0 \%)$ & $8(53,3 \%)$ \\
$\quad$ Masculino & $9(60,0 \%)$ & $12(80,0 \%)$ \\
Feminino & $6(40,0 \%)$ & $3(20,0 \%)$ \\
\hline Espaços & $7(46,6 \%)$ & $12(80,0 \%)$ \\
A & $7(46,6 \%)$ & $3(20,0 \%)$ \\
B & $1(6,8 \%)$ & $0(0,0 \%)$ \\
C & $8(53,3 \%)$ & $8(53,3 \%)$ \\
\hline Forame & $7(46,7 \%)$ & $7(46,7 \%)$ \\
FTF (fissura transforme) & & \\
FPF (fissura pré-forame) & &
\end{tabular}

\section{DISCUSSÃO}

No século XX até a década de 1940, pouco se conhecia sobre a dinâmica facial e o surgimento do apoio cefalométrico criou novas perspectivas para os fissurados em geral. Todavia, a partir de $1950^{7}$, os enxertos começam a ser preconizados para prevenir o colapso maxilar, sem, entretanto, ficar definido qual a melhor idade para sua feitura ${ }^{4}$, pois até os sete anos ele inevitavelmente afetará o crescimento maxilar que se faz por aposição ${ }^{8}$. Ao lado disto, há a erupção dentária como fator a ser considerado, sendo que atualmente a idade preconizada como a mais indicada é dos nove aos 12 anos ${ }^{6}$, apesar de não haver unanimidade $9,10,11$. 
Quanto à nossa experiência (Quadro 1), no grupo A (enxerto autólogo) seis pacientes (40\%) estavam entre nove12 anos, enquanto que no grupo B sete $(46,7 \%)$ pertenciam ao mesmo intervalo etário, sendo equivalente a localização das FLP em relação ao forame incisivo para os dois grupos. Quanto ao sucesso dos enxertos aferidos pelos espaços A, sete $(46,6 \%)$ e $12(80,0 \%)$, respectivamente, foram encontrados para os grupos A e B, sendo importante salientar que a idade média do grupo B foi de 13,2 anos e do A 16,2 anos. Vale portanto inferir que quanto mais próxima a idade do intervalo nove- 12 anos maior a chance do sucesso, apesar de tê-lo alcançado em pacientes com até 21 anos de idade. A comparação destes achados com a literatura mundial, permite compreender a necessidade de se estabilizar o maxilar através do enxerto ósseo no adolescente ${ }^{9}$. Ao lado disto, o reconhecimento da importância da indicação do enxerto antes da erupção dos caninos ${ }^{5,6}$, torna este intervalo etário o mais indicado para o procedimento, apesar de reconhecermos as dificuldades da não aceitação de pacientes fora da mesma em nosso meio, onde o retardo da procura de centros especializados é regra.

Quanto ao estabelecimento de critérios avaliativos do sucesso dos enxertos 7 , vários parâmetros foram enumerados:

1. Fechamento da mucosa nasal com retalho de fundo de saco gengival e labial livre de tensão, através de deslizamento simples ou oblíquo, plastia em $\mathrm{Z}$ ou retalho palatino complementar, sendo que alguns autores sugerem a inclusão do perióstio para promover a osteogênese $^{5,8,11,12}$, aumentando ainda a mobilidade do retalho e diminuindo a tensão.

2. Para a oclusão da cavidade bucal, a reconstrução do plano mucoso é irrelevante, pois depende do enxerto ósseo utilizado isolado ou associado ao material aloplástico a partir da crista ilíaca. Nesta última eventualidade, o emprego da hidroxiapatita permite a manutenção de matriz óssea por um período maior do que enxerto puro, diminuindo sua absorção e aumentando a osteogênese. Estes fatores ficam bem demonstrados nos resultados alcançados ( $80 \%$ de sucesso para o enxerto e hidroxiapatita e $46,7 \%$ para o enxerto puro).

3. A avaliação dos resultados através de imagens passa por uma extensa discussão, onde a radiografia periapical convencional ${ }^{13,14,15,16}$ era contraposta à panorâmica ${ }^{17} \mathrm{e}$ à tomografia computadorizada ${ }^{18,19}$.

A radiografia digital pode ser uma alternativa interessante que permite a análise da imagem instantaneamente, o armazenamento em disquetes, menor distorção em relação à panorâmica e menor custo em relação à tomografia computadorizada. O sistema por nós utilizado permite avaliar a densidade das imagens para o enxerto e para o osso no prazo de três meses. Com uma magnificência de $2 \%$, possibilita a feitura de gráficos sobre a imagem, determinando três espaços para avaliação dos resultados dos enxertos puros ou associados. O sucesso é a localização da maior concavidade do enxerto no espaço A (entre as linhas 1 - traçada na região cervical do dente adjacente à fissura com menor distorção - e 2, que dista $3 \mathrm{~mm}$ da primeira. Quanto ao insucesso, este é aferido pela localização da maior concavidade do enxerto nos espaços B e C (entre as linhas 2, 3 e 4). Em síntese, o tratamento cirúrgico do FLP deve ser feito entre os nove e 12 anos de idade, através do enxerto autólogo de crista ilíaca triturado e associado à material aloplástico (hidroxiopatita), sendo a radiografia digital o método de escolha da integração do enxerto ósseo pós-tratamento cirúrgico.

\begin{abstract}
The reconstruction of alveolar process in cleft lip palate patients is mandatory to enable the eruption and movement of permanent teeth through the clefted area by orthodontic procedure, beside the establisment of aesthetics with prostetic procedure by osteointegrated implants. Considering that autogenous bone grafts provide a high reabsortion level determining a reduction of orthodontic movement or making impossible to place implants, to minimize this problem, we developed a protocol by which the bone (anterior iliac crest) is mixed with alloplastic material (hydroxyapatite) to keep the bone matrix framework, reducing the absotion degree at the clefted area. We selected 30 patients at the Centro de Atendimento Integral ao Fissurado Lábio-Palatino (Center of Integral Care of Cleft Lip and Palate Patients), in Curitiba, Paraná (from 1997 to 1999). The patients were divided in two groups: at the first one, we utilized grated autogenous iliac crest and in the last one, the same bone combined with bio-reabsorbable hydroxyapatite. After 6 months, we compared for both procedures, the results through digital periapical X ray, concerning the height of the alveolar bone of the adjacent teeth, and 4 limiting lines for bone reconstruction as follow: line 1 was drawn at the cervical region of the adjacent tooth to the less distorted cleft and lines 2,3 and 4, parallel to line 1, being 3mm apart from each other. It was successfull considered the grafts whose greatest concavity was between lines 1 and 2 (space A) and for grafts between lines 2 and 3 (space B) or 3 and 4 (space C), surgical failure. The control group (grated bone) had 46,7\% of the patients in space A while the study group (bone plus alloplastic material) $80 \%$ of patients in this space, being the determining factor of sucess the patient age.
\end{abstract}

Key Words: Bone; Graft; Autogenous 


\section{REFERENCIAS}

1. Ellis III, E.. Management of patients with orofacial clefts. In: Contemporary Oral and Maxillofacial Surgery. Peterson, L..J.; Ellis III,E.; Hupp,J.R. et al.. $2^{\circ}$ ed. St.Louis: Mosby - Year Book, Inc.,1988, p.657-681.

2. Sicher, H. \& Tandler, J. Anatomia para Dentistas. Ed. Atheneu, São Paulo,1981, p.283-309.

3. Spina,V. A proposed modification for the classification of cleft lip and palate. Cleft Palate J. , 1973, 10:25122516.

4. Witsenburg, B. The Reconstruction of Anterior Residual Bone Defects in patients with Cleft Lip, Alveolus and Palate. J. Max-Fac. Surg., 1985, 13:197-208.

5. Boyne, P. J. \& Sands, N.R. Secondary bone grafting of residual alveolar and palated clefts. J. Oral Surg, 1972, 30:87-92.

6. Boyne, P.J. Osseous Reconstruction of the Maxilla and the Mandible. Surgical Techniques Using Titanium Mesh and Bone Mineral. Editora Quintessence books, 1997, cap. 1 e 2, p. 3-15.

7. Shafer, D. M. Secondary Bone Grafting for Unilateral Alveolar Clefts: A Review of Surgical Techniques. Oral Max Surg N Amer, 1995, 1:29-42.

8. Enemark, H.; Pedersen-Sindet, S. ; Bundgaard, M. LongTerm Results after Secondary Bone Grafting of Alveolar Clefts. J.Oral Maxillofac.Surg., 1987, 45:913918.

9. Paulin,G. ; Astrand, P. ; Rosenquist et al. Intermediate Bone Grafting of Alveolar Clefts. J. Cranio-Max.- Fac. Surg., 1988, 16:2-7.

10. Kortebein,M. J.; Nelson, C. L. ; Sadove, M. A. . Retrospective Analysis of 135 Secondary Alveolar Cleft Grafts Using Iliac or Calvarial Bone. J. Oral Maxillofac. Surg., 1991, 49:493-498.

11. Amanat,N. \& Langdon, J. D. Secondary Alveolar Bone Grafting in Clefts of the Lip and Palate. J. CranioMax.Fac.Surg., 1991, 19:7-14.

12. Schultz,R. C. . Free Periosteal Graft Repair of Maxillary Clefts in Adolescents. Plastic and Reconstructive Surgery., 1984, 73:556-563.
13. Takahashi,T.; Fukuda,M. ; Yamaguchi,T. et al.. Use of Endosseous Implants for Dental Reconstruction of Patients with Grafted Alveolar Clefts. J. Oral Maxillofac. Surg., 1997, 55:576-583.

14. Koole,R.; Bosker,H ; Van Der Dussen, N. Late Secondary Autogenous Bone Grafting in Cleft Patients Comparing Mandibular (Ectomesenchymal) and Iliac Crest (Mesenchymal) Grafts. J. Cranio-Max-Surg., 1989, 17:28-30.

15. Turvey,T. A. ; VIG, K. ; Moriarty et al.. Delayed bone Grafting in the Cleft Maxilla and Palate: A Retrospective Multidisciplinary Analysis. Am.J. Orthod., 1984, 86:244-256.

16. Pedersen-Sindet, S. \& Enemark, H. Mandibular Bone Grafts for Reconstruction of Alveolar Clefts. J. Oral Maxillofac. Surg., 1988, 46:533-537.

17. Akesson,L..; Hakansson,J ; Rohlin,MA.. Comparison of Panoramic and Intraoral Radiography and pocket probing for the measurement of the marginal bone level. J. Clin. Periodontal ., 1992, 19:326-332.

18. Van Der Meij, A. J.; Baart, B.P.; Valk,J.; et al.. Computed Tomography in Evaluation of Early Secondary Bone Grafting. Int. J. Oral Maxillofac. Surg., 1994, 23:132136.

19. Lee,C.; Crepeau,R. J.; Williams, H.B. et al.. Alveolar Cleft Bone Grafts: Results and Imprecisions of the Dental Radiograph. Plast and Reconst Surg, 1995, 96:15341538 .

Endereço para correspondência:

Prof. Dr. Abrão Rapoport

Pça. Amadeu Amaral, 47 - cj. 82 - Paraíso

01327-010 São Paulo - SP

E-mail: cpgcp.hosphel@attglobal.net 